\title{
Editorial
}

Psychotherapy and Psychosomatics
Psychother Psychosom 2011;80:195-198

DOI: $\underline{10.1159 / 000324510}$
Received: January 15, 2011

Accepted after revision: January 24, 2011

Published online: April 14, 2011

\section{Clinical Factor 2010}

\author{
Richard Balon \\ Department of Psychiatry and Behavioral Neurosciences and Department of Anesthesiology, \\ Wayne State University School of Medicine, Detroit, Mich., USA
}

In his editorial 'The clinical factor' [1], Giovanni Fava announced that we will try to select papers published in psychiatric journals during the previous year that I would subjectively perceive as having a high 'Clinical Factor' the degree and extent to which a journal article provides information to the clinician that may improve his/her practice.

Thus, I am presenting my subjective selection of 15 articles which I believe may provide some information to help the reader improve his/her clinical practice. Many may find them useful, some will find them less useful or useless. I am certainly biased, as is anybody else. I also cannot review all psychiatric journals - I focused only on most of the major ones. If there is no article selected from a certain major psychiatric journal, it does not necessarily mean that I have not reviewed it or skimmed through that journal. It may mean that I did not find, in my opinion, anything clinically very interesting. I reviewed the following journals: American Journal of Psychiatry, Archives of General Psychiatry, British Journal of Psychiatry, Depression and Anxiety, European Psychiatry, Journal of Affective Disorders, Journal of Clinical Psychiatry, Journal of Clinical Psychopharmacology, Journal of Sex and Marital Therapy, Psychological Medicine, and Psychotherapy and Psychosomatics. I view myself first and foremost as a clinician (though academically oriented) and I am, as most clinicians are, looking for a way to help patients, and thus looking for new, or innovative, or newly validated treatments. That is part of my clinician bias and that impacts my selection - almost all articles are on treatment issues.

Those who know me and know that I am basically a clinical psychopharmacologist may be surprised that the selection below contains only a minority of articles on 'new' medications and articles presenting the results of psychopharmacology trials. Psychopharmacology has brought us a great many advances in the past, but, in my humble opinion, not much lately. As I do not believe that an umptieth comparison of two medications with minuscule differences in efficacy or side effects represents much clinical news, advance or something that may significantly improve one's clinical practice, such comparisons are not included.

Last but not least, this selection is not a competition, there are no winners. The articles are listed in references [2-16] alphabetically, not by any order of importance, preference or significance. Here is the selection:

The first discussed article by Bosanac et al. [2] is actually an editorial review that discusses early intervention in psychotic disorders. The authors warn us that the early psychosis movement, however well-intentioned, has overreached itself, setting dogma before service. The authors point out that the evidence indicating benefits from early intervention are sustained and meaningful in the

\section{KARGER}

Fax +41 613061234

E-Mail karger@karger.ch

www.karger.com (c) 2011 S. Karger AG, Basel

0033-3190/11/0804-0195\$38.00/0

Accessible online at:

www.karger.com/pps
Richard Balon, MD

UPC-Jefferson

2751 E. Jefferson \# 200

Detroit, MI 48207 (USA)

Tel. +1 313993 3416, E-Mail rbalon@wayne.edu 
longer term is equivocal. Thus, at the present time, we should allocate resources equally for all patients with psychosis. (There was no funding for this editorial review.)

The results of the study by Byers et al. [3] showed that the short-term effectiveness of prazosin and quetiapine for nighttime PTSD symptoms was similar; however, patients prescribed prazosin were significantly more likely to continue treatment to the end of the study than those prescribed quetiapine. In addition, patients treated with quetiapine discontinued the medication because of adverse effects more frequently. The authors suggested that prazosin should be used as first-line for nighttime PTSD symptoms in veterans. This is certainly a wise clinical suggestion. (There was no funding for this VA study.)

The article by Ingehoven et al. [4] is a meta-analysis of pharmacotherapy studies of personality disorders. The authors concluded that drug therapy tailored to well-defined symptom domains can have an effect on patients with severe personality disorders. The authors also questioned the current pharmacological algorithms based on the results of their meta-analysis. According to their findings, antipsychotics had a moderate effect on cognitive-perceptual symptoms and a moderate-tolarge effect on anger. Surprisingly, antidepressants had no effect on impulsive-behavioral dyscontrol and global function, but had a small effect on anxiety and anger. Finally, interestingly, mood stabilizers had a very large effect on impulsive-behavioral dyscontrol and anger, a large effect on anxiety and a moderate effect on depression and global function (more than antipsychotics in this domain). These results may certainly change our approach to specific pharmacotherapy of severe personality disorders. (Funding for this study was provided by The Netherlands Expertise Center for Forensic Psychiatry.)

Lancee et al. [5] reported that imagery rehearsal therapy and exposure were equally effective in ameliorating nightmares. They suggested that exposure to nightmare imagery may be a crucial factor, but cognitive restructuring may be added to increase the immediate effect. The important fact is that this self-help approach was more cost-effective when compared to face-to-face intervention. Self-help approaches such as this one could be a great and inexpensive remedy for patients with nightmares. (Funding for this study was provided by the Dutch Foundation for Mental Health, Amersfoort, The Netherlands.)

The review article by Loo et al. [6] reviewed augmenting strategies for difficult-to-treat electroconvulsive therapy patients. Interestingly, according to the authors' lit- erature review, concurrent administration of some antidepressants may be helpful. Other helpful strategies included reduction of treatment frequency to twice per week, hyperventilation before each treatment, and use of remifentanil to minimize the dosage of induction anesthetics with anticonvulsant properties. These recommendations may help in devising treatment strategies for these difficult to treat patients. (This review was partially funded by an Australian National Health and Medical Research Council project grant.)

Another meta-analysis, this time by Lynch et al. [7], pointed out that cognitive-behavioral therapy (CBT) is no better than non-specific control intervention in the treatment of schizophrenia and that it does not reduce relapse. The analysis suggested that though CBT is effective in major depression, the size of the effect is small in analyzed treatment studies. Finally, CBT is not an effective strategy for relapse prevention in bipolar disorder. These results will probably help to reduce the indiscriminant use of CBT in these three major psychiatric disorders. (This meta-analysis was supported by The Instituto de Salud Carlos III, CIBERSAM, Barcelona, Spain.)

An interesting study by Maina et al. [8] demonstrated that supplementary brief dynamic therapy had no significant effect on both obsessive and depressive symptomatology in patients with obsessive-compulsive disorder and major depressive disorder who were receiving effective medication. These results should guide us not to use additional brief dynamic therapy in obsessive compulsive and depressed patients who do well on their medication, and thus reduce the cost of treatment. (No information about the funding for this study was provided.)

Martens et al. [9] found a robust association between generalized anxiety disorder and cardiovascular events in outpatients with coronary heart disease. This association could not be explained by disease severity, health behavior or biological mediators. The traditional focus on depression in patients with coronary heart disease should also be expanded to generalized anxiety disorder. It would be interesting to see whether the treatment of concurrent generalized anxiety disorder would lead to better outcomes of coronary heart disease. (This study was supported by the VA Epidemiology Program; VA Health Service Research and Development Service Career Development Program; grant from the National heart, Lung, and Blood Institute; American Federation for Aging Research; Robert Wood Johnson Foundation; Ischemia Research and Education Foundation, and the Nancy Kirwan Heart Research Fund.) 
Moss-Morris et al. [10] in their study of primary care patients with irritable bowel syndrome found out that CBT-based self-management in the form of a structured manual with minimal therapist control was effective and acceptable in these patients (even at 8 months). Thus, selfmanagement CBT based strategies may be a helpful intervention in irritable bowel syndrome, a fairly treatmentresistant condition. (Funding for this study was provided by the University of Auckland Staff Fund, Auckland, New Zealand.)

In another study of CBT in physical illness, NavarreteNavarrete et al. [11] demonstrated that CBT was effective in helping patients suffering from lupus erythematosus with high levels of stress. CBT helped to reduce stress, anxiety and depression in these patients and improved their quality of life. These results suggest that using CBT in serious physical illness could be quite helpful in alleviating various psychological symptoms and thus CBT should be used in this population. (This study was funded by the Andalusian Health System, Spain.)

Modafinil was effective and well tolerated in treating fatigue in HIV+ patients in a study by Rabkin et al. [12]. Patients treated with modafinil had more energy and less depressive symptomatology than those on placebo, even after 6 months. Modafinil had minimal side effects and was well accepted by the patients. Modafinil could be a new and useful addition to our armamentarium of treatments of symptoms associated with HIV. (The authors reported no funding for this study; they are supported by the New York State Psychiatric Institute, New York, N.Y., USA.)

Segal et al. [13] reported that mindfulness-based cognitive therapy (8 weekly group sessions) offered protection against relapse/recurrence on par of that of maintenance pharmacotherapy in depressed patients achieving stable or unstable remission. They also emphasized the importance of maintaining at least one long-term active treatment in unstable remitters. Mindfulness-based CBT may be another option for maintenance treatment of remitted depressed patients, which may be easy and costeffective to implement. (This study was supported by a grant form the National Institute of Mental Health, Bethesda, Md., USA.)

Patients with bulimia nervosa who failed to report a $\geq 60 \%$ decrease in binge eating or vomiting at week 3 of treatment were unlikely to respond to fluoxetine in a study by Sysko et al. [14]. As no pretreatment characteristic was helpful in predicting response, early response to treatment with fluoxetine may be the only available indicator to guide clinical management of bulimia nervosa.
(This study was supported by the Rahr Eating Disorder Research Project Fund, and some data were provided by Eli Lilly Company.)

The results of a study by Wilson et al. [15] indicated that interpersonal psychotherapy (20 sessions in 6 months) and guided self-help based on CBT (10 sessions in 6 months) were significantly more effective than behavioral weight loss treatment in eliminating binge eating after 2 years. The authors recommend that guided self-help based on CBT should be the first-line option for most patients with binge eating disorder, with interpersonal or full CBT used for patients with low self-esteem and high eating disorder psychopathology. These psychotherapy modalities are another enrichment of our armamentarium for treatment of binge eating. (This study was funded by grants from the National Institutes of Health, Bethesda, Md., USA.)

Finally, Young et al. [16] reported that Interpersonal Psychotherapy-Adolescent Skills Training (IPT-AST) leads to an immediate reduction in depressive symptoms and improved overall functioning in adolescents with elevated depressive symptomatology. However, the benefits were not consistent beyond the 6-month follow-up. The authors thus suggested that the preventive effect was limited and the effectiveness of possible booster sessions of IPT-AST should be studied. The IPT-AST would be another treatment option for depressed adolescents that may be easy to implement. Booster sessions in every CBT variation could be helpful and should be studied. (There was no funding reported for this study, but the authors indicated no financial relationships to disclose in the previous 3 years.)

\section{References}

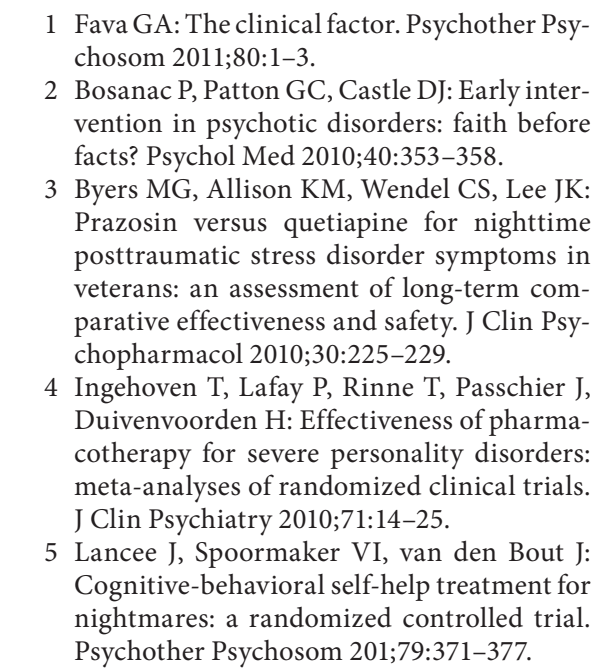

Psychother Psychosom 2011;80:195-198 
6 Loo CK, Kaill A, Paton P, Simpson B: The difficult-to-treat electroconvulsive therapy patient - strategies for augmenting outcomes. J Affect Disord 3010;124:219-227.

7 Lynch D, Laws KR, McKenna PJ: Cognitive behavioral therapy for major psychiatric disorder: does it really work? A meta-analytical review of well-controlled trials. Psychol Med 2010;40:9-24

-8 Maina G, Rosso G, Rigardetto S, Chiado-Piat S, Bogetto F: No effect of adding brief dynamic therapy to pharmacotherapy in the treatment of obsessive-compulsive disorder with concurrent major depression. Psychother Psychosom 2010;79:295-302.

-9 Martens EJ, de Jonge P, Na B, Cohen BE, Lett H, Whooley MA: Scared to death? Generalized anxiety disorder and cardiovascular events in patients with stable coronary heart disease: The Heart and Soul Study. Arch Gen Psychiatry 2010;67:750-758.
10 Moss-Morris R, McAlpine L, Didsbury LP, Spence MJ: A randomized controlled trial of a cognitive behavioural therapy-based self-management intervention for irritable bowel syndrome in primary care. Psychol Med 2010;40:85-94

11 Navarrete-Navarrete N, Peralta-Ramirez MI, Sabio-Sanchez JM, Coin MA, RoblesOrtega H, Higaldo-Tenorio C, Ortego-Centeno N, Callejas-Rubio JL, Jimenez-Alonso J: Efficacy of cognitive behavioural therapy for the treatment of chronic stress in patients with lupus erythematosus: a randomized controlled trial. Psychother Psychosom 2010;79:107-115.

12 Rabkin JG, McElhiney MC, Rabkin R, Mc Grath PJ: Modafinil treatment for fatigue in HIV/AIDS: a randomized placebo-controlled trial. J Clin Psychiatry 2010;71:707-715.
3 Segal ZV, Bieling P, Young T, MacQueen G, Cooke R, Martin L, Bloch R, Levitan RD: Antidepressants monotherapy vs sequential pharmacotherapy and mindfulness-based cognitive therapy, or placebo, for relapse prophylaxis in recurrent depression. Arch Gen Psychiatry 2010;67:1256-1264.

14 Sysko R, Sha N, Wang Y, Duan N, Walsh BT: Early response to antidepressants treatment in bulimia nervosa. Psychol Med 2010;40: 999-1005.

15 Wilson GT, Wilfley DE, Agras WS, Bryson SW: Psychological treatments of binge eating disorder. Arch Gen Psychiatry 2010;67:94101.

16 Young JF, Mufson L, Gallop R: Preventing depression: a randomized trial of interpersonal therapy-adolescent skills training. Depress Anxiety 2010;27:426-433. 